\title{
QUASILINEAR SYSTEMS WITH SEVERAL PERIODIC SOLUTIONS ${ }^{1}$
}

\author{
JANE CRONIN
}

Abstract. By using topological degree, it is proved that for a certain class of quasilinear systems of ordinary differential equations of the form

$$
\dot{x}=A(t) x+\epsilon \mu f(x, t, \mu)+\mu g(x, t, \mu)+h(t)
$$

where $\epsilon, \mu$ are small parameters and $A, f, g, h$ are periodic in $t$, there exist at least two periodic solutions.

The classical method for studying the resonance case of the problem of finding periodic solutions of quasilinear systems of ordinary differential equations is to derive a system of bifurcation equations and apply the implicit function theorem to this system (Coddington and Levinson [1], [2, Chapter 14]). An extension of this method is to use topological degree instead of the implicit function theorem. If the degree is nonzero, there is at least one periodic solution; moreover by using Sard's theorem and varying certain of the terms in the equation arbitrarily slightly, it can be shown that the degree is a lower bound for the number of periodic solutions of the varied system [3, Chapter II]. Here we study the problem of estimating the number of solutions in the case that the topological degree may be zero. We will show that for the special class of quasilinear systems

$$
\dot{x}=A(t) x+\epsilon \mu f(x, t, \mu)+\mu g(x, t, \mu)+h(t)
$$

where $\epsilon, \mu$ are small parameters, the linear part of $f$ in $x$ is well behaved and $g$ is essentially an even function (the precise hypotheses on (E) will be described later), there are at least two solutions of (E).

We assume throughout that the following conditions are satisfied on $(\mathrm{E})$.

(1) The elements of matrix $A(t)$ and the components of $f, g, h$ are defined for all real $t$ and have continuous first derivatives in $t$ for all $t$.

(2) For all $t$, the components of $f$ and $g$ are defined for $\mu$ in an interval containing $\mu=0$ and for all $x$ in real Euclidean $n$-space. The

Received by the editors September 21, 1970.

AMS 1970 subject classifications. Primary 34C25, 55C25.

Key words and phrases. Topological degree, quasilinear systems of ordinary differential equations, periodic solutions.

${ }_{1}^{1}$ The research in this paper was supported by the U. S. Army Research Office (Durham) under Grant DA-ARO-31-124-G1098.

Copyright (0) 1971. American Mathematical Society 
components of $f$ and $g$ have continuous first derivatives in $\mu$ and in the components of $x$.

(3) Matrix $A(t)$ and functions $f, g, h$ have period $T$ in $t$.

Let $M(t)$ be a fundamental matrix of $\dot{x}=A(t) x$ such that $M(0)$ is the identity matrix. If $x(t, \mu, \epsilon, c)$ is a solution of (E) that satisfies boundary condition $c$ at $t=0$, i.e., if $x(0, \mu, \epsilon, c)=c$, then a necessary and sufficient condition that $x(t, \mu, \epsilon, c)$ have period $T$ in $t$ is that $c$ satisfy the equation

$$
\begin{aligned}
& \begin{array}{ll}
{[M(T)-M(0)] c+M(T) \int_{0}^{T}[M(s)]^{-1}\{\epsilon \mu f[x(s, \mu, \epsilon, c), s, \mu]} \\
(1) & +\mu g[x(s, \mu, \epsilon, c), s, \mu]+h(s)\} d s \\
\quad=0 . &
\end{array}
\end{aligned}
$$

(See [3, Chapter II ].) We assume complete resonance, i.e., we assume $M(T)-M(0)=0$. (Our study is applicable to the case where the resonance is not complete, i.e., where $0<\operatorname{rank}[M(T)-M(0)]<n$, but this case is more complicated and involves no new principles. So we use the stronger hypothesis of complete resonance.) First a necessary condition that (1) can be solved for $c$ as function of $\mu$ in neighborhoods of $c=0$ and $\mu=0$ for a fixed $\epsilon$ is that

$$
\int_{0}^{T}[M(s)]^{-1} h(s) d s=0 .
$$

See $[3$, p. 69]. So we assume from now on that (2) holds. Thus (1) becomes

$$
\begin{aligned}
& \epsilon \mu \int_{0}^{T}[M(s)]^{-1} f[x(s, \mu, \epsilon, c), s, \mu] d s \\
& +\mu \int_{0}^{T}[M(s)]^{-1} g[x(s, \mu, \epsilon, c), s, \mu] d s=0 .
\end{aligned}
$$

Dividing by $\mu$ and letting $\mu \rightarrow 0$ in the resulting equation, we obtain

$$
\begin{aligned}
\epsilon \int_{0}^{T}[M(s)]^{-1} f[x(s, 0, \epsilon, c), s, 0] d s \\
+\int_{0}^{T}[M(s)]^{-1} g[x(s, 0, \epsilon, c), s, 0] d s=0 .
\end{aligned}
$$

Note that $x(s, 0, \epsilon, c)=x(s, 0,0, c)$. Hence (4) becomes: 


$$
\begin{aligned}
\epsilon \int_{0}^{T}[M(s)]^{-1} f[x(s, 0,0, c), s, 0] d s \\
+\int_{0}^{T}[M(s)]^{-1} g[x(s, 0,0, c), s, 0] d s=0 .
\end{aligned}
$$

Regard $\epsilon$ as fixed. Then in order to determine whether (3) can be solved for $c$ as a function of $\mu$ in neighborhoods of $c=0$ and $\mu=0$, we study the topological degree of the mapping of $c$-space (i.e., real Euclidean $n$-space) into itself described by the left side of (5). Let

$$
\epsilon \int_{0}^{T}[M(s)]^{-1} f[x(s, 0,0, c), s, 0] d s=\epsilon F(c)
$$

and

$$
\int_{0}^{T}[M(s)]^{-1} g[x(s, 0,0, c), s, 0] d s=G(c) .
$$

Also let $U$ be a bounded open set in $c$-space. We denote the Brouwer degree of a mapping $A$ relative to $\bar{U}$ at the point $p$ by $\operatorname{deg}(A, \bar{U}, p)$.

Lemma. If $\operatorname{deg}(G, \bar{U}, 0)$ exists, then if $|\epsilon|$ is sufficiently small, $\operatorname{deg}(\epsilon F+G, \bar{U}, 0)$ exists and equals $\operatorname{deg}(G, \bar{U}, 0)$.

Proof. Follows from the definition of degree and the invariance under homotopy of the degree.

Theorem 1. If $\operatorname{deg}(G, \bar{U}, 0)$ is odd, there is an $\epsilon_{0}>0$ such that if $\epsilon_{1}$ is any fixed real number with $\left|\epsilon_{1}\right|<\epsilon_{0}$, then there is a $\delta\left(\epsilon_{1}\right)$ such that for all $\mu$ with $|\mu|<\delta\left(\epsilon_{1}\right)$, the equation (E) with $\epsilon=\epsilon_{1}$ has a periodic solution.

Proof. By the lemma, if $|\epsilon|$ is sufficiently small then $\operatorname{deg}(\epsilon F+G, \bar{U}, 0)$ is odd and hence nonzero. The theorem follows from the derivation of (3), (4) and (5).

Now suppose $F(c)=L(c)+R(c)$ where $L(c)$ is linear in $c$ and $\lim _{|c| \rightarrow 0}(R(c) /|c|)=0$. Suppose $L(c)$ is a nonsingular linear map so that $\operatorname{deg}(\epsilon L, \bar{B}, 0)= \pm 1$ where $\bar{B}$ is a closed ball with center 0 and arbitrary radius.

Theorem 2. If $\lim _{|c| \rightarrow 0}(G(c) /|c|)=0$ and $\operatorname{deg}(G, \bar{U}, 0)$ is defined and is even, then for each fixed sufficiently small $\epsilon_{1}$, there is a $\delta\left(\epsilon_{1}\right)$ such that equation (E) with $\epsilon=\epsilon_{1}$ has for each $\mu$ with $|\mu|<\delta\left(\epsilon_{1}\right)$ at least two distinct solutions.

Proof. For $\epsilon$ sufficiently small,

$$
\operatorname{deg}(\epsilon L+\epsilon R+G, \bar{U}, 0)=\operatorname{deg}(G, \bar{U}, 0)=\text { even number. }
$$


Take a fixed sufficiently small $\epsilon$. Since for any $\bar{B}, \operatorname{deg}(\epsilon L, \bar{B}, 0)$ $= \pm 1$ and since $\lim _{|c| \rightarrow 0}(R(c) /|c|)=0$ and $\lim _{|c| \rightarrow 0}(G(c) /|c|)=0$, it follows that if $\bar{B}$ has a sufficiently small radius, then

$$
\operatorname{deg}(\epsilon L+\epsilon R+G, \bar{B}, 0)=\operatorname{deg}(\epsilon L, \bar{B}, 0)= \pm 1 \text {. }
$$

But by a basic property of the degree,

$$
\begin{aligned}
\operatorname{deg}(\epsilon L+\epsilon R & +G, \overline{U-\bar{B}}, 0) \\
& =\operatorname{deg}(\epsilon L+\epsilon R+G, \bar{U}, 0)-\operatorname{deg}(\epsilon L+\epsilon R+G, \bar{B}, 0) .
\end{aligned}
$$

By (6) and (7), the expression on the right in (8) is a number different from zero. Thus equation (3) has a collection of solutions $c_{1}(\mu)$ (at least one for each $\mu$ with $|\mu|<\delta(\epsilon)$ ) such that each $c_{1}(\mu)$ is in $U-\bar{B}$. By (7), equation (3) has a collection of solutions $c_{2}(\mu)$ such that each $c_{2}(\mu)$ is in $\bar{B}$. This completes the proof of the theorem.

CoRollary. If $\lim _{|c| \rightarrow 0}(G(c) /|c|)=0$ and $G$ is an even function and $\operatorname{deg}(G, \bar{U}, 0)$ is defined, the conclusion of Theorem 2 holds.

Proof. If $G$ is even then $\operatorname{deg}(G, \bar{U}, 0)$ is even by Krasnosel'skiY [4, p. 223, footnote].

EXAMPLE. Let the components of $f(x, t, 0)$ and $g(x, t, 0)$ be polynomials in the components of $x$ with coefficients which are periodic (of period $T$ ) functions of $t$ and let $\bar{U}$ be a closed ball $\bar{B}_{1}$ with center at the origin. Then we can apply Theorem 1 if the components of $g$ are odd polynomials and apply Theorem 2 if the components of $g$ are even polynomials whose lowest order terms are all of degree $\geqq 2$ and if $\epsilon L(c)$ is nonsingular. To make the application, it is necessary to show that $\operatorname{deg}\left(G, \bar{B}_{1}, 0\right)$ is defined. One situation in which this can be done is the following: if the components of $g(x, t, 0)$ are homogeneous polynomials all of the same degree, then the components of $G$ are homogeneous polynomials $G_{1}, G_{2}, \cdots, G_{n}$ all of the same degree, and $\operatorname{deg}\left(G, \bar{B}_{1}, 0\right)$ is defined if the resultant of $G_{1}, \cdots, G_{n}$ is nonzero.

REMARKS. 1. It is natural to raise the question of whether the collections $c_{1}(\mu), c_{2}(\mu)$ which appear in the proof of Theorem 2 define continuous functions of $\mu$. The collection $c_{2}(\mu)$ is a continuous function because in applying (7) one is actually applying the implicit function theorem. But the collection $c_{1}(\mu)$ may be much more complicated. (It might happen, for example, that for some value of $\mu$, there is an infinite set of solutions of (3).)

2. Results parallel to the above can be obtained by parallel arguments for the equation 


$$
x=A(t) x+\mu f(x, t, \mu)+M \mu g(x, t, \mu)
$$

where $\mu$ is a small parameter and $M$ is a constant such that $|M|$ is sufficiently large.

\section{BiBLIOGRAPHY}

1. E. A. Coddington and N. Levinson, Perturbations of linear systems with constant coefficients possessing periodic solutions, Contributions to the Theory of Nonlinear Oscillations, vol. 2, Princeton Univ. Press, Princeton, N. J., 1952, pp. 19-35. MR 14, 981.

2. - Theory of ordinary differential equations, McGraw-Hill, New York, 1955. MR 16, 1022.

3. J. Cronin, Fixed points and topological degree in nonlinear analysis, Math. Surveys, no. 11, Amer. Math. Soc., Providence, R. I., 1964. MR 29 \#1400.

4. M. A. Krasnosel'skir, Topological methods in the theory of non-linear integral equations, GITTL, Moscow, 1956; English transl., Macmillan, New York, 1964. MR 20 \#3464; MR 28 \#2414.

Rutgers University, New Brunswick, New Jersey 08903 